\title{
Alkylation of phenylglycinol-derived bicyclic lactams. Enantioselective synthesis of 3-alkylpiperidines
}

\author{
Mercedes Amat,* Carmen Escolano, Núria Llor, Oscar Lozano, Arantxa Gómez-Esqué, \\ Rosa Griera, and Joan Bosch*
}

Laboratory of Organic Chemistry, Faculty of Pharmacy, University of Barcelona, Av. Joan XXIII s/n, Barcelona 08028, Spain

E-mail: amat@ub.edu ; joanbosch@ub.edu

\author{
This work is dedicated to Professors José Elguero and Pedro Molina \\ (received 08 Nov 04; accepted 10 Feb 05; published on the web 02 Mar 05)
}

\begin{abstract}
The stereochemical outcome of the alkylation of simple phenylglycinol-derived bicyclic lactams has been studied. The method provides a simple and concise route to 3-alkylpiperidines in both enantiomeric series. The synthesis of $(+)$-R-decarbomethoxytetrahydrosecodine, an indole alkaloid embodying a 3-ethylpiperidine moiety, is reported.
\end{abstract}

Keywords: Chiral bicyclic lactams, phenylglycinol, alkylation, enantioselective synthesis

\section{Introduction}

Chiral non-racemic bicyclic -lactams formed by cyclo-condensation of simple -oxoesters and $(R)$ - or $(S)$-phenylglycinol have emerged as powerful materials for easy access to a variety of enantiopure substituted piperidines by stereoselective introduction of the substituents on the ring, taking advantage of the functionalization and conformational rigidity of the bicyclic lactam system.1 In this context, we have published a preliminary report 2 that alkylation of the enolate derived from the lactam carbonyl takes place with high facial stereoselectivity to give, ultimately, enantiopure 3-alkylpiperidines.

Although alkylation at the position $\alpha$ - to the carbonyl group of bicyclic $\gamma$ - and $\delta$ - lactams derived from phenylglycinol or other chiral aminoalcohols has received considerable attention, both from the synthetic and theoretical standpoint, ${ }^{3}$ the origin of the facial stereoselectivity remains controversial and the observed stereoselectivities are difficult to rationalize. We report here a short and convenient route for the synthesis of enantiopure 3-alkylpiperidines based on the alkylation of simple phenylglycinol-derived bicyclic lactams, cis-1 and trans-1, and illustrate the potential and usefulness of this approach with the enantioselective synthesis of $(+)$ Rdecarbomethoxytetrahydrosecodine, an indole containing a 3-ethylpiperidine moiety, and its enantiomer. 


\section{Results and Discussion}

The pure lactam (-)-cis-1 is easily accessible by cyclo-condensation of $(R)$-phenylglycinol with methyl 5-oxopentanoate under neutral conditions, followed by column chromatography of the resulting 85:15 diastereomeric mixture of lactams, while the lactam (-)-trans-1 is obtained by equilibration of the above mixture under acidic conditions followed by chromatographic purification. $^{1 \mathrm{a}, 4}$

The enolate of the lactam (-)-trans-1 was initially generated by treatment with LDA. Subsequent alkylation with methyl iodide or benzyl bromide gave the corresponding exo 3substituted 2-piperidones (-)-2 and (+)-3 with good stereoselectivity (only one diastereomer was observed by NMR) but only moderate chemical yield (44\% and $26 \%$, respectively). ${ }^{5}$ This low yield can be attributed to the fact that LDA removes the benzylic methine proton of (-)-trans-1, with irreversible opening of the oxazolidine ring to give an $N$-styryl lactam. ${ }^{6}$ In fact, singlets at $\delta$ 5.3 and 5.7, attributable to the vinyl protons, were observed in the ${ }^{1} \mathrm{H}-\mathrm{NMR}$ spectrum of the crude reaction mixtures. The above yields were improved to $77 \%$ and $50 \%$, respectively, when LiHMDS [lithium bis(trimethylsilyl)amide] was used as the base. Under these conditions, alkylation of (-)-trans-1 with ethyl iodide gave (-)-4 in 83\% yield, also with excellent exo facial stereoselectivity. Only one diastereoisomer was observed by NMR in the crude reaction mixture (Scheme 1).

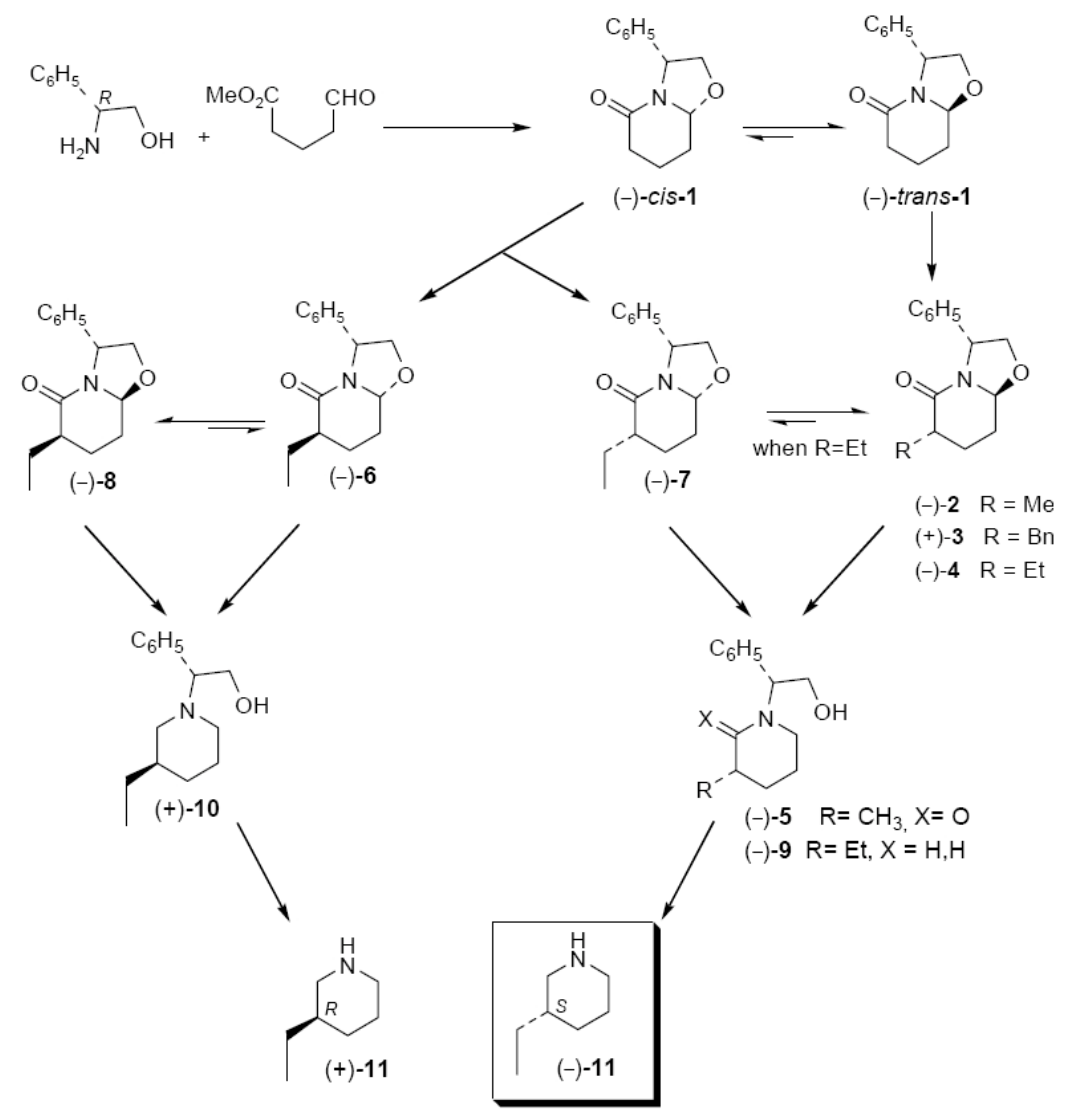

\section{Scheme 1}


The configuration of the new stereogenic center in the alkylated lactams was determined by X-ray diffraction analysis of the ethyl lactam (-)-4, and by reducing the bicyclic lactam (-)-2 to the known ${ }^{7}(\alpha R, 3 S)$ - hydroxylactam (-)-5, whose configuration had previously been determined by X-ray analysis.

In contrast with the above satisfactory results, alkylation (LiHMDS) of the lactam (-)-cis-1 with ethyl iodide took place with moderate stereoselectivity to give a 1:2 diastereomeric mixture of exo- and endo- lactams (-)-6 and (-)-7 in 77\% overall yield. The configuration of the stereocenter (C-6) generated in the above alkylation was determined by equilibration experiments. Thus, treatment of the major endo epimer (-)-7 with TFA in $\mathrm{CH}_{2} \mathrm{Cl}_{2}$ led to the previously prepared lactam (-)-4 [(-)-4/(-)-7 in a 13:1 ratio], whereas the minor exo epimer (-)-6 was converted to a 2:1 mixture of a new lactam (-)-8 and (-)-6.

Treatment of lactam (-)-4 with $\mathrm{LiAlH}_{4}$ brought about both the reduction of the lactam carbonyl group and reductive cleavage of the oxazolidine ring to give the piperidine (-)-9. A similar reduction of (-)-7 led to the same piperidine (-)-9, thus confirming that (-)-4 and (-)-7 are epimers at the methine 8a- carbon. Finally, removal of the chiral auxiliary by hydrogenolysis in the presence of $\mathrm{Pd} / \mathrm{C}$ gave $(S)$-3-ethylpiperidine (-)-11 in 76\% yield. By following a similar sequence, the minor epimeric lactams (-)-6 and (-)-8 were converted to piperidine $(+)-\mathbf{1 0}$ and then to $(R)$-3-ethylpiperidine $(+)-\mathbf{1 1}$.

A more convenient access to 3-alkylpiperidines in the $R$ - enantiomeric series simply involves starting from $(S)$-phenylglycinol, which is also commercially available. Thus, the bicyclic lactam $(+)$-trans-1 was alkylated stereoselectively to $(+)-4$ and then converted in excellent yield to $(R)$ 3-ethylpiperidine $(+)-\mathbf{1 1}$ via the piperidine $(+)-9$ (Scheme 2; see Experimental Section). The above approach provides a simple and concise route to 3-alkylpiperidines in both enantiomeric series. It is worth mentioning that, with only one recent exception, ${ }^{8}$ both $(R)$ - and $(S)-3$ ethylpiperidine have been obtained previously by resolution of the racemate. 9

To illustrate the potential of chiral lactams 1, the enantiopure piperidines $(+)-\mathbf{1 1}$ and $(-)-\mathbf{1 1}$ were alkylated with 3-(2-bromoethyl)-2-ethylindole ${ }^{2 b}$ to give, respectively the alkaloid $(+)$ decarbomethoxytetrahydrosecodine and its enantiomer. ${ }^{10}$

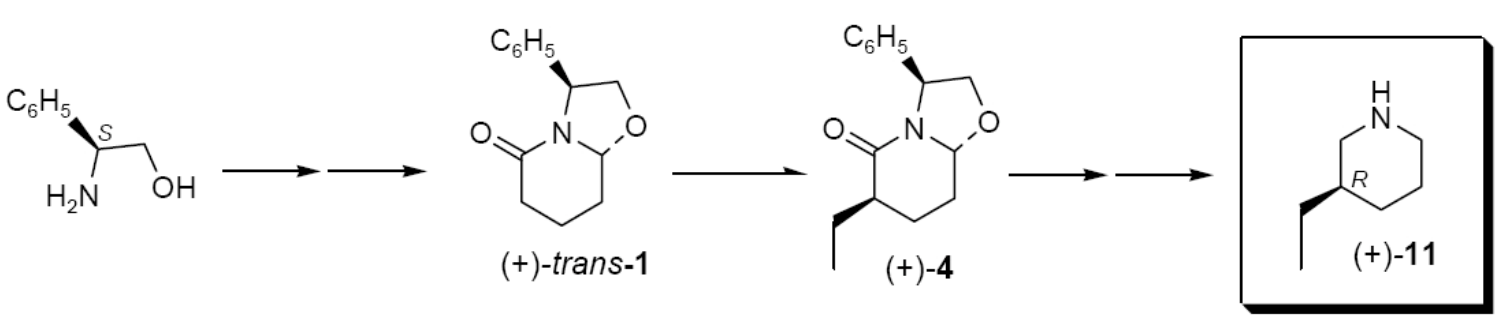

\section{Scheme 2}




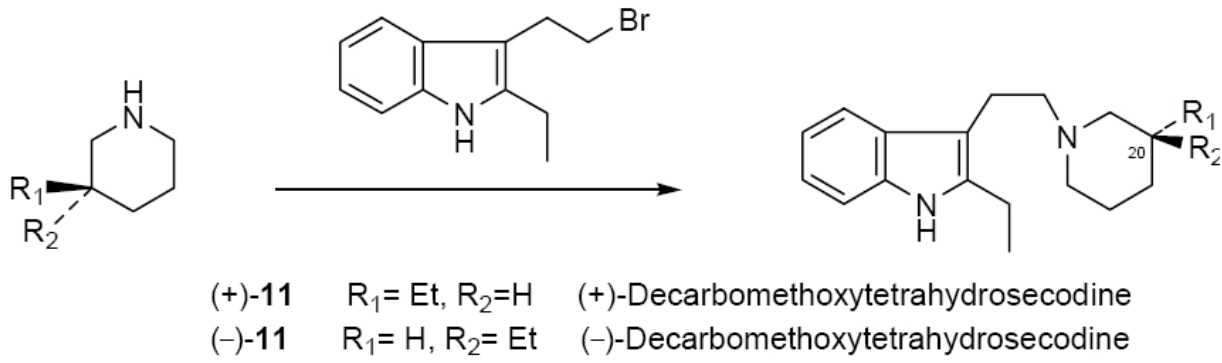

Scheme 3

\section{Experimental Section}

General Procedures. All reactions were performed under an argon or nitrogen atmosphere with dry, freshly distilled solvents using standard procedures. Drying of organic extracts during the work-up of reactions was performed over anhydrous $\mathrm{Na}_{2} \mathrm{SO}_{4}$ or $\mathrm{MgSO}_{4}$. Evaporation of solvents was accomplished with a rotary evaporator. Thin-layer chromatography used $\mathrm{SiO}_{2}$ (silica gel 60 F254), and the spots were located by UV and either a $1 \% \mathrm{KMnO}_{4}$ solution or iodine. Chromatography refers to flash column chromatography and was carried out on $\mathrm{SiO}_{2}$ (silica gel 60, SDS, 230-400 mesh). Melting points were determined in a capillary tube and are uncorrected. Unless otherwise indicated, NMR spectra were recorded in $\mathrm{CDCl}_{3}$. The chemical shifts are reported as $\delta$ values, in parts per million (ppm) relative to $\mathrm{Me}_{4} \mathrm{Si}(0 \mathrm{ppm})$ or relative to residual chloroform (7.26 ppm, $77.0 \mathrm{ppm})$ as an internal standard. Data are reported in the following manner: chemical shift, multiplicity, integrated intensity, coupling constant $(J)$ in Hertz $(\mathrm{Hz})$ and assignment (when possible). Multiplicities are reported using the following abbreviations: s, singlet; d, doublet; t, triplet; q, quartet; m, multiplet; br, broad; ap, apparent. Assignments and stereochemical determinations are given only when they are derived from definitive two-dimensional NMR experiments (HMQC-COSY). Only noteworthy IR absorptions $\left(\mathrm{cm}^{-1}\right)$ are listed. Mass spectra (MS) data are reported as $\mathrm{m} / \mathrm{z}(\%)$. High-resolution mass spectra (HMRS) were performed in the Unidade de Espectrometria de Masas, Santiago de Compostela. Microanalyses were performed by the Centre d'Investigació i Desenvolupament (CSIC), Barcelona.

\section{General procedure for the alkylation of lactams}

cis-1 and trans-1. A solution of $\mathbf{1}(1 \mathrm{mmol})$ in THF was added to a cooled $\left(-78{ }^{\circ} \mathrm{C}\right)$ solution of LiHMDS ( $1 M$ in THF, $1.5 \mathrm{mmol}$ ) in THF. After stirring the solution at $-78{ }^{\circ} \mathrm{C}$ for $1 \mathrm{~h}$, the alkylating reagent $(2.7 \mathrm{mmol})$ was added and stirring was continued for an additional $2 \mathrm{~h}$. The reaction was quenched by addition of saturated aqueous $\mathrm{NaCl}$, and the resulting mixture was extracted with EtOAc and $\mathrm{CH}_{2} \mathrm{Cl}_{2}$. The combined organic extracts were dried and concentrated, and the resulting residue was chromatographed.

(3R,6S,8aS)-6-Methyl-5-oxo-3-phenyl-2,3,6,7,8,8a-hexahydro-5H-oxazolo[3,2-a]pyridine [(-)-2]. The lactam, (-)-trans-1 (600 mg, $2.76 \mathrm{mmol})$ in THF (6 mL), LiHMDS (4.14 mL, $4.14 \mathrm{mmol})$ in THF (24 mL), and methyl iodide $(0.44 \mathrm{~mL}, 7.05 \mathrm{mmol})$ afforded $(-)-2(491 \mathrm{mg}, 77 \%)$ after flash chromatography $(2: 3$ 
EtOAc-hexane): IR ( $\mathrm{NaCl}) 1648 \mathrm{~cm}^{-1} ;{ }^{1} \mathrm{H}-\mathrm{NMR}\left(\mathrm{CDCl}_{3}, 300 \mathrm{MHz}, \mathrm{COSY}\right.$, HETCOR) $\delta 1.25$ (d, J = 7.2 Hz, 3H, CH $), 1.44-1.62$ (m, 2H, H-7, H-8), 2.03 (m, 1H, H-7), 2.31-2.41 (m, 2H, H6, H-8), 3.73 (dd, $J=9.0,8.1 \mathrm{~Hz}, 1 \mathrm{H}, \mathrm{H}-2), 4.48$ (dd, $J=9.0,8.4 \mathrm{~Hz}, 1 \mathrm{H}, \mathrm{H}-2), 5.01$ (dd, $J=$ 8.7, $4.5 \mathrm{~Hz}, 1 \mathrm{H}, \mathrm{H}-8 \mathrm{a}$ ), 5.24 (app t, $J=8.1 \mathrm{~Hz}, 1 \mathrm{H}, \mathrm{H}-3), 7.21-7.32$ (m, 5H, H-Ar); ${ }^{13} \mathrm{C}-\mathrm{NMR}$ $\left(\mathrm{CDCl}_{3}, 75.4 \mathrm{MHz}\right) \delta 18.2\left(\mathrm{CH}_{3}\right), 26.0(\mathrm{C}-7), 28.2(\mathrm{C}-8), 37.0(\mathrm{C}-6), 58.0(\mathrm{C}-3), 72.7(\mathrm{C}-2), 88.7$ (C-8a), 125.7 (2C, Ar), 127.3 (C, Ar), 128.6 (2C, Ar), 139.5 (C, ipso), $172.0(\mathrm{NCO})$; $[\alpha]^{22} \mathrm{D}-$ 104.7 (c 1.0 MeOH). Anal. Calcd for $\mathrm{C}_{14} \mathrm{H}_{17} \mathrm{NO}_{2}$ : C, 72.70; H, 7.41; N, 6.06. Found C, 72.31; H, $7.46 ; \mathrm{N}, 5.97 \%$.

(3R,6R,8aS)-6-Benzyl-5-oxo-3-phenyl-2,3,6,7,8,8a-hexahydro-5H-oxazolo[3,2-a]pyridine

[(+)-3]. The lactam, (-)-trans-1 (1 g, $4.61 \mathrm{mmol})$ in THF $(15 \mathrm{~mL})$, LiHMDS $(6.92 \mathrm{~mL}, 6.92$ $\mathrm{mmol})$ in THF $(35 \mathrm{~mL})$, and benzyl bromide $(1.41 \mathrm{~mL}, 2.35 \mathrm{mmol})$ afforded $(+)-3(711 \mathrm{mg}$, $50 \%)$ after flash chromatography (1:9 EtOAc-hexane): IR (NaCl) $1653 \mathrm{~cm}^{-1} ;{ }^{1} \mathrm{H}-\mathrm{NMR}\left(\mathrm{CDCl}_{3}\right.$, $300 \mathrm{MHz}, \mathrm{COSY}, \mathrm{HETCOR}) \delta 1.45-1.53$ (m, 2H, H-7, H-8), 1.81 (m, 1H, H-7), 2.28 (m, 1H, H8), 2.62 (m, 1H, H-6), 2.94 (dd, $J=13.5,8.0 \mathrm{~Hz}, 1 \mathrm{H}, \mathrm{CH}_{2} \mathrm{Ph}$ ), 3.16 (dd, $J=13.5,4.0 \mathrm{~Hz}, 1 \mathrm{H}$, $\mathrm{CH}_{2} \mathrm{Ph}$ ), 3.66 (dd, $\left.J=9.0,8.0 \mathrm{~Hz}, 1 \mathrm{H}, \mathrm{H}-2\right), 4.47$ (dd, 9.0, 8.0 Hz, 1H, H-2), 4.84 (dd, $J=8.5$, $5.0 \mathrm{~Hz}, 1 \mathrm{H}, \mathrm{H}-8 \mathrm{a}), 5.26$ (app t, $J=7.8 \mathrm{~Hz}, 1 \mathrm{H}, \mathrm{H}-3$ ), 7.10-7.36 (m, 10H, H-Ar); ${ }^{13} \mathrm{C}-\mathrm{NMR}$ $\left(\mathrm{CDCl}_{3}, 75.4 \mathrm{MHz}\right) \delta 22.1(\mathrm{C}-7), 28.0(\mathrm{C}-8), 38.0\left(\mathrm{CH}_{2} \mathrm{Ph}\right), 43.4(\mathrm{C}-6), 58.3(\mathrm{C}-3), 72.8(\mathrm{C}-2)$, 88.7 (C-8a), 125.9 (2C Ar), 126.1 (C Ar), 127.4 (C Ar), 128.1 (2C Ar), 128.6 (2C Ar), 129.1 (2C Ar), 138.7 (C ipso Bn), 139.3 (C ipso Ph), 170.5 (NCO); $[\alpha]^{22} \mathrm{D}+26.5$ (c 1.0 MeOH); MS-EI m/z $307\left(\mathrm{M}^{+}, 95\right), 104$ (100), 216 (72); HRMS calcd for $\mathrm{C}_{20} \mathrm{H}_{21} \mathrm{NO}_{2} 307.1572$, found 307.1569.

(3R,6S,8aS)-6-Ethyl-5-oxo-3-phenyl-2,3,6,7,8,8a-hexahydro-5H-oxazolo-[3,2-a]-pyridine [()-4]. The lactam, (-)-trans-1 (200 mg, $0.92 \mathrm{mmol})$ in THF ( $2 \mathrm{~mL})$, LiHMDS (1.38 mL, 1.38 $\mathrm{mmol})$ in THF $(8 \mathrm{~mL})$, and ethyl iodide $(0.19 \mathrm{~mL}, 2.36 \mathrm{mmol})$ afforded (-)-4 (187 mg, 83\%) after flash chromatography (1:1 EtOAc-hexane): IR ( NaCl) $1640 \mathrm{~cm}^{-1} ;{ }^{1} \mathrm{H}-\mathrm{NMR}\left(\mathrm{CDCl}_{3}, 300\right.$ $\mathrm{MHz}, \mathrm{COSY}$, HETCOR) $\delta 0.93\left(\mathrm{t}, J=7.5 \mathrm{~Hz}, 3 \mathrm{H}, \mathrm{CH}_{3} \mathrm{CH}_{2}\right), 1.50-1.60(\mathrm{~m}, 2 \mathrm{H}, \mathrm{H}-7, \mathrm{H}-8), 1.66$ $\left(\mathrm{m}, 1 \mathrm{H}, \mathrm{CH}_{3} \mathrm{CH}_{2}\right), 1.90\left(\mathrm{~m}, 1 \mathrm{H}, \mathrm{CH}_{3} \mathrm{CH}_{2}\right), 2.04(\mathrm{~m}, 1 \mathrm{H}, \mathrm{H}-7), 2.32(\mathrm{~m}, 1 \mathrm{H}, \mathrm{H}-6), 2.40(\mathrm{~m}, 1 \mathrm{H}$, $\mathrm{H}-8$ ), 3.73 (dd, $J=9.0,8.0 \mathrm{~Hz}, 1 \mathrm{H}, \mathrm{H}-2), 4.51$ (dd, $J=9.0,8.0 \mathrm{~Hz}, 1 \mathrm{H}, \mathrm{H}-2), 5.02$ (dd, $J=8.6$, $4.8 \mathrm{~Hz}, 1 \mathrm{H}, \mathrm{H}-8 \mathrm{a}), 5.28$ (t, $J=8.0 \mathrm{~Hz}, 1 \mathrm{H}, \mathrm{H}-3), 7.20-7.38(\mathrm{~m}, 5 \mathrm{H}, \mathrm{ArH}) ;{ }^{13} \mathrm{C}-\mathrm{NMR}\left(\mathrm{CDCl}_{3}\right.$, $75.4 \mathrm{MHz}) \delta 10.7\left(\mathrm{CH}_{3} \mathrm{CH}_{2}\right), 22.2(\mathrm{C}-7), 25.4\left(\mathrm{CH}_{3} \mathrm{CH}_{2}\right), 28.1(\mathrm{C}-8), 42.8(\mathrm{C}-6), 58.2(\mathrm{C}-3), 72.7$ (C-2), 88.8 (C-8a), 125.7 (C Ar), 127.4 (C Ar), 128.7 (C Ar), 139.7 (C ipso), 171.7 (NCO); mp 90-92 ${ }^{\circ} \mathrm{C}\left(\mathrm{Et}_{2} \mathrm{O}-\right.$ hexane); $[\alpha]^{22} \mathrm{D}-103.0$ (c $\left.1.0 \mathrm{EtOH}\right) ; \mathrm{MS}-\mathrm{EI} \mathrm{m} / \mathrm{z} 245\left(\mathrm{M}^{+}, 25\right), 55$ (88), 104 (100). Anal. Calcd for $\mathrm{C}_{15} \mathrm{H}_{19} \mathrm{NO}_{2}: \mathrm{C}, 73.44 ; \mathrm{H}, 7.81 ; \mathrm{N}, 5.71$. Found: C, 73.29; H, 7.87; N, $5.70 \%$.

$(3 S, 6 R, 8 a R)-6-E t h y l-5-0 x 0-3-p h e n y l-2,3,6,7,8,8 a-h e x a h y d r o-5 H-0 x a z o l o[3,2-a] p y r i d i n e ~[(+)-$ 4]. Following the general procedure, the lactam (+)-trans-1 gave (+)-4: $m p$ 91-92 ${ }^{\circ} \mathrm{C} ;[\alpha]^{22} \mathrm{D}$ +102.9 (c $1.0 \mathrm{EtOH}$ ). Anal. Calcd for $\mathrm{C}_{15} \mathrm{H}_{19} \mathrm{NO}_{2}$ : C, 73.44; H, 7.81; N, 5.71. Found: C, 73.76; $\mathrm{H}, 7.81 ; \mathrm{N}, 5.52 \%$.

$(3 R, 6 R, 8 \mathrm{a} R)$ - and (3R,6S,8aR)-6-Ethyl-5-oxo-3-phenyl-2,3,6,7,8,8a-hexahydro-5H-oxazolo[3,2-a]pyridine [(-)-6 and (-)-7]. The lactam, (-)-cis-1 (400 mg, $1.84 \mathrm{mmol})$ in THF (4 mL), LiHMDS $(2.76 \mathrm{~mL}, 2.76 \mathrm{mmol})$ in THF $(18 \mathrm{~mL})$, and ethyl iodide $(0.37 \mathrm{~mL}, 4.59 \mathrm{mmol})$ afforded (-)-7 (234 mg, 52\%) and (-)-6 (112 mg, 25\%) after flash chromatography (1:1 EtOAc- 
hexane). (-)-7: ${ }^{1} \mathrm{H}-\mathrm{NMR}\left(\mathrm{CDCl}_{3}, 300 \mathrm{MHz}, \mathrm{COSY}, \mathrm{HETCOR}\right) \delta 0.90(\mathrm{t}, J=7.5 \mathrm{~Hz}, 3 \mathrm{H}$, $\left.\mathrm{CH}_{3} \mathrm{CH}_{2}\right), 1.44\left(\mathrm{~m}, 1 \mathrm{H}, \mathrm{CH}_{3} \mathrm{CH}_{2}\right), 1.74\left(\mathrm{~m}, 1 \mathrm{H}, \mathrm{CH}_{3} \mathrm{CH}_{2}\right), 1.80-1.96$ (m, 3H, 2H-7, H-8), 2.16 (m, 1H, H-6), 2.27 (m, 1H, H-8), 3.99 (dd, $J=9.0,1.2 \mathrm{~Hz}, 1 \mathrm{H}, \mathrm{H}-2), 4.15$ (dd, $J=9.0,6.8 \mathrm{~Hz}$, $1 \mathrm{H}, \mathrm{H}-2), 4.86$ (dd, $J=8.7,3.8 \mathrm{~Hz}, 1 \mathrm{H}, \mathrm{H}-8 \mathrm{a}), 4.90$ (dd, $J=6.8,1.2 \mathrm{~Hz}, 1 \mathrm{H}, \mathrm{H}-3), 7.19-7.35$ $(\mathrm{m}, 5 \mathrm{H}, \mathrm{ArH}) ;{ }^{13} \mathrm{C}-\mathrm{NMR}\left(\mathrm{CDCl}_{3}, 75.4 \mathrm{MHz}\right) \delta 11.9\left(\mathrm{CH}_{3} \mathrm{CH}_{2}\right), 21.8(\mathrm{C}-7), 24.7\left(\mathrm{CH}_{3} \mathrm{CH}_{2}\right), 25.5$ (C-8), 41.2 (C-6), 58.4 (C-3), 73.9 (C-2), 88.2 (C-8a), 126.1 (C Ar), 127.2 (C Ar), 128.3 (C Ar), 141.6 (C- ipso), 170.3 (NCO); $[\alpha]^{22}{ }_{\mathrm{D}}-52.0$ (c $\left.0.5 \mathrm{EtOH}\right)$. Anal. Calcd for $\mathrm{C}_{15} \mathrm{H}_{19} \mathrm{NO}_{2}$ : C, 73.44; $\mathrm{H}, 7.81$; N, 5.71. Found C, 73.31; H, 8.03; N, 5.51\%. (-)-6: ${ }^{1} \mathrm{H}-\mathrm{NMR}\left(\mathrm{CDCl}_{3}, 300 \mathrm{MHz}, \mathrm{COSY}\right.$, HETCOR) $\delta 0.91\left(\mathrm{t}, J=7.5 \mathrm{~Hz}, 3 \mathrm{H}, \mathrm{CH}_{3} \mathrm{CH}_{2}\right), 1.50\left(\mathrm{~m}, 2 \mathrm{H}, \mathrm{CH}_{3} \mathrm{CH}_{2}, \mathrm{H}-7\right), 1.80$ (m, 1H, H-8a), 1.90 (m, $1 \mathrm{H}, \mathrm{CH}_{3} \mathrm{CH}_{2}$ ), 2.06-2.23 (m, 2H, H-6, H-7), 2.42 (ddd, $J=12.3,6.9,3.3 \mathrm{~Hz}, 1 \mathrm{H}, \mathrm{H}-8$ ), 4.02 (dd, $J=9.0,1.2 \mathrm{~Hz}, 1 \mathrm{H}, \mathrm{H}-2), 4.16$ (dd, $J=9.0,6.8 \mathrm{~Hz}, 1 \mathrm{H}, \mathrm{H}-2), 4.83$ (dd, $J=9.9,3.3$

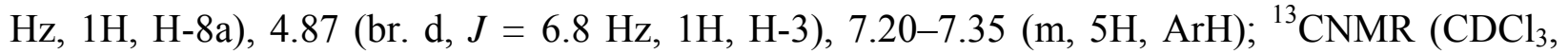
$75.4 \mathrm{MHz}) \delta 11.0\left(\mathrm{CH}_{3} \mathrm{CH}_{2}\right), 23.3(\mathrm{C}-7), 24.2\left(\mathrm{CH}_{3} \mathrm{CH}_{2}\right), 28.2(\mathrm{C}-8), 42.4(\mathrm{C}-6), 59.0(\mathrm{C}-3), 73.8$ (C-2), 88.6 (C-8a), 126.2 (C Ar), 127.2 (C Ar), 128.3 (C Ar), 141.6 (C- ipso), 169.4 (NCO); mp $104-106{ }^{\circ} \mathrm{C}\left(\mathrm{Et}_{2} \mathrm{O}-\right.$ hexane $) ;[\alpha]^{22} \mathrm{D}-100.0$ (c $\left.0.93 \mathrm{EtOH}\right)$. Anal. Calcd for $\mathrm{C}_{15} \mathrm{H}_{19} \mathrm{NO}_{2}$ : C, 73.44; H, 7.81; N, 5.71. Found C, 73.33; H, 7.85; N, 5.60\%.

$(3 S, 6 S, 8 a S)-$ and (3S,6R,8aS)-6-Ethyl-5-oxo-3-phenyl-2,3,6,7,8,8a-hexahydro-5H-oxazolo[3,2-a]pyridine [(+)-6 and (+)-7]. Following the general procedure, lactam $(+)$-cis-1 gave (+)-6 and (+)-7. (+)-6: mp 104-105 ${ }^{\circ} \mathrm{C}\left(\mathrm{Et}_{2} \mathrm{O}-\right.$ hexane $) ;[\alpha]^{22} \mathrm{D}+103.2(c 1.0 \mathrm{EtOH}) .(+)-7:[\alpha]^{22} \mathrm{D}+$ 53.7 ( с $0.5 \mathrm{EtOH})$.

Equilibration of (-)-6 and (-)-7. TFA (1 mL) was added to a solution of (-)-6 (315 mg, $1.29 \mathrm{mmol})$ in $\mathrm{CH}_{2} \mathrm{Cl}_{2}(25 \mathrm{~mL})$ and the mixture was stirred at $\mathrm{RT}$ for $40 \mathrm{~h}$. Then $\mathrm{CH}_{2} \mathrm{Cl}_{2}$ was added, and the mixture was washed with saturated aqueous $\mathrm{NaHCO}_{3}$ and water. The organic phase was dried and concentrated to afford (-)-6 and (-)-8 (1:2 mixture; determined by ${ }^{1} \mathrm{H}-$ NMR). Column chromatography (1:1 EtOAc-hexane) afforded (-)-8: ${ }^{1} \mathrm{H}-\mathrm{NMR}\left(\mathrm{CDCl}_{3}\right.$, $300 \mathrm{MHz}$, COSY, HETCOR) $\delta 1.00\left(\mathrm{t}, J=7.4 \mathrm{~Hz}, 3 \mathrm{H}, \mathrm{CH}_{3}\right), 1.49\left(\mathrm{~m}, 1 \mathrm{H}, \mathrm{CH}_{2}\right), 1.60-2.05(\mathrm{~m}$, $4 \mathrm{H}, \mathrm{CH}_{3} \mathrm{CH}_{2}, \mathrm{H}-7, \mathrm{H}-8_{\text {ax }}$ ), 2.23 (m, 2H, H-6, H- $8_{\text {eq }}$ ), 3.77 (dd, J = 8.9, $\left.7.7 \mathrm{~Hz}, 1 \mathrm{H}, \mathrm{H}-2\right), 4.47$ $(\mathrm{dd}, J=8.8,8.1 \mathrm{~Hz}, 1 \mathrm{H}, \mathrm{H}-2), 5.01(\mathrm{dd}, J=7.7,4.7 \mathrm{~Hz}, 1 \mathrm{H}, \mathrm{H}-8 \mathrm{a}), 5.26(\mathrm{t}, J=7.9 \mathrm{~Hz}, 1 \mathrm{H}, \mathrm{H}-$ 3), 7.20-7.38 (m, 5H, ArH); ${ }^{13} \mathrm{C}-\mathrm{NMR}\left(\mathrm{CDCl}_{3}, 50 \mathrm{MHz}\right) \delta 12.2\left(\mathrm{CH}_{3}\right), 20.7(\mathrm{C}-7), 24.0$ $\left(\mathrm{CH}_{3} \mathrm{CH}_{2}\right), 25.4$ (C-8), 41.4 (C-6), 58.1 (C-3), 72.2 (C-2), 88.2 (C-8a), 126.0 (C Ar), 127.3 (C Ar), 128.6 (C Ar), 139.6 (C ipso), 171.7 (NCO); mp $63-64{ }^{\circ} \mathrm{C}$; $[\alpha]^{22} \mathrm{D}-156.4$ (c $\left.0.5 \mathrm{EtOH}\right)$. Anal. Calcd for $\mathrm{C}_{15} \mathrm{H}_{19} \mathrm{NO}_{2}$ : C, 73.44; H, 7.81; N, 5.71. Found C, 73.42; H, 7.89; N, 5.73\%. From lactam (+)-6, (+)-8 was obtained: $\mathrm{mp} 64-65{ }^{\circ} \mathrm{C}$; $[\alpha]^{22} \mathrm{D}+154.0\left(\right.$ c $\left.0.5 \mathrm{CHCl}_{3}\right)$. From (-)-7 as described above, a mixture of (-)-7 and (-)-4 (1:13 ratio; determined by $1 \mathrm{H}-\mathrm{NMR})$ was obtained.

(3S)- $N$-[(1R)-2-Hydroxyethyl-1-phenyl]-3-methyl-2-piperidone (-)-5. Triethylsilane $(0.04 \mathrm{~mL}, 0.2 \mathrm{mmol})$ and $\mathrm{TiCl}_{4}(0.02 \mathrm{~mL}, 0.3 \mathrm{mmol})$ were added to a cooled solution $\left(-78{ }^{\circ} \mathrm{C}\right)$ of (-)-2 (30 mg, $0.2 \mathrm{mmol}$ ) in $\mathrm{CH}_{2} \mathrm{Cl}_{2}$. The mixture was allowed to warm to RT, stirred for $8 \mathrm{~h}$, and poured into saturated aqueous $\mathrm{NH}_{4} \mathrm{Cl}$. The aqueous phase was extracted with $\mathrm{CH}_{2} \mathrm{Cl}_{2}$. The combined organic extracts were dried and concentrated to give a residue, which was 
chromatographed (EtOAc) to afford (-)-5 (21 mg, 70\%): IR (NaCl) 3406, $1618 \mathrm{~cm}^{-1}$; ${ }^{1} \mathrm{H}-\mathrm{NMR}$ $\left(\mathrm{CDCl}_{3}, 300 \mathrm{MHz}, \mathrm{COSY}, \mathrm{HETCOR}\right) \delta 1.28\left(\mathrm{~d}, J=7.2 \mathrm{~Hz}, 3 \mathrm{H}, \mathrm{CH}_{3} \mathrm{CH}\right), 1.45(\mathrm{~m}, 1 \mathrm{H}, \mathrm{H}-4)$, 1.73 (m, 2H, H-5), 1.96 (m, 1H, H-4), 2.52 (m, 1H, H-3), 2.89 (ddd, $J=12.1,7.4,5.8 \mathrm{~Hz}, 1 \mathrm{H}$, H-6), 3.18 (ddd, $J=12.1,6.2,6.2 \mathrm{~Hz}, 1 \mathrm{H}, \mathrm{H}-6$ ), 3.40 (br. s, $1 \mathrm{H}, \mathrm{OH}$ ), 4.13 (m, 2H, $\mathrm{CH}_{2} \mathrm{OH}$ ), $5.78(\mathrm{dd}, J=8.8,5.5,1 \mathrm{H}, \mathrm{NCHAr}), 7.25-7.33(\mathrm{~m}, 5 \mathrm{H}, \mathrm{ArH}) ;{ }^{13} \mathrm{C}-\mathrm{NMR}\left(\mathrm{CDCl}_{3}, 75.4 \mathrm{MHz}\right) \delta$ $18.2\left(\mathrm{CH}_{3}\right), 21.3$ (C-5), 28.7 (C-4), 36.7 (C-3), 43.7 (C-6), 58.5 (NCHAr), $61.7\left(\mathrm{CH}_{2} \mathrm{OH}\right), 127.6$ (C Ar), 127.7 (C Ar), 128.5 (C Ar), 137.0 (C- ipso), 175.4 (NCO); MS-EI m/z 234 (M+1, 100), $216(26), 114(50) ;[\alpha]^{22}{ }^{-} 70.0\left(c 0.7 \mathrm{CHCl}_{3}\right)$.

\section{General procedure for $\mathrm{LiAlH}_{4}$ reduction}

$\mathrm{LiAlH}_{4}(500 \mathrm{mg}, 13.2 \mathrm{mmol})$ was added in portions to a solution of lactams 4, 6, 7, or 8 (1 g, $4.08 \mathrm{mmol})$ in THF $(50 \mathrm{~mL})$. The mixture was stirred at RT for $1 \mathrm{~h}$. Then $15 \%$ aqueous $\mathrm{NaOH}$ was carefully added, the resulting suspension was filtered, and the residue was washed with $\mathrm{Et}_{2} \mathrm{O}$. The combined organic extracts were concentrated to give (902 $\left.\mathrm{mg}, 95 \%\right)$ of the respective piperidines, 9 (from 4 or 7 ) or 10 (from 6 or 8).

(3S)-3-Ethyl-1-[(1R)-2-hydroxy-1-phenylethyl]piperidine [(-)-9]. ${ }^{1} \mathrm{H}-\mathrm{NMR}\left(\mathrm{CDCl}_{3}, 300\right.$ MHz, COSY, HETCOR) $\delta 0.71(\mathrm{qd}, J=11.6,3.0 \mathrm{~Hz}, 1 \mathrm{H}, \mathrm{H}-4), 0.88\left(\mathrm{t}, J=7.3 \mathrm{~Hz}, 3 \mathrm{H}, \mathrm{CH}_{3}\right.$ ), 1.19 (m, 2H, $\mathrm{CH}_{3} \mathrm{CH}_{2}$ ), 1.43-1.53 (m, 2H, H-3, H-5), 1.57-1.75 (m, 3H, H-4, H-5, H-6), 1.95 (t, $J=10.5 \mathrm{~Hz}, 1 \mathrm{H}, \mathrm{H}-2), 2.79$ (m, 2H, H-2, H-6), 3.40 (br. s, $1 \mathrm{H}, \mathrm{OH}), 3.60$ (dd, 1H, $J=10.2,5.1$ Hz, H-2'), 3.70 (dd, $J=10.2,5.1 \mathrm{~Hz}, 1 \mathrm{H}, \mathrm{H}-1$ '), 3.98 (t, $J=10.2 \mathrm{~Hz}, 1 \mathrm{H}, \mathrm{H}-2$ '), $7.12-7.40$ (m, $5 \mathrm{H}, \mathrm{ArH}) ;{ }^{13} \mathrm{C}-\mathrm{NMR}\left(\mathrm{CDCl}_{3}, 50 \mathrm{MHz}\right) \delta 11.3\left(\mathrm{CH}_{3}\right), 25.1(\mathrm{C}-5), 26.9\left(\mathrm{CH}_{3} \mathrm{CH}_{2}\right), 30.2(\mathrm{C}-4)$, 38.0 (C-3), 47.2 (C-6), 58.6 (C-2), 60.0 (C-2'), 70.3 (C-1'), 128.1 (C- p), 128.2 (C-o), 129.1 (Cm), 134.7 (C-ipso); $[\alpha]^{22}{ }_{\mathrm{D}}-27.2$ (c $\left.0.5 \mathrm{EtOH}\right)$. Anal. Calcd for $\mathrm{C}_{15} \mathrm{H}_{23} \mathrm{NO}$ : C, 77.20; H, 9.94; N, 6.00. Found C, 77.13; H, 7.97; N, 6.00\%. (+)-9: $[\alpha]^{22}{ }_{\mathrm{D}}+28.1($ c $0.5 \mathrm{EtOH})$.

(3R)-3-Ethyl-1-[(1R)-2-hydroxy-1-phenylethyl]piperidine [(+)-10]. ${ }^{1} \mathrm{H}-\mathrm{NMR}\left(\mathrm{CDCl}_{3}, 300\right.$ MHz, COSY, HETCOR) $\delta 0.63(\mathrm{~m}, 1 \mathrm{H}, \mathrm{H}-4), 0.77\left(\mathrm{t}, J=7.3 \mathrm{~Hz}, 3 \mathrm{H}, \mathrm{CH}_{3}\right), 1.07(\mathrm{~m}, 2 \mathrm{H}$, $\mathrm{CH}_{3} \mathrm{CH}_{2}$ ), 1.29 (m, 2H, H-2, H-3), 1.43-1.68 (m, 3H, H-4, H-5), 2.17 (td, $J=11.2,3.4 \mathrm{~Hz}, 1 \mathrm{H}$, H- 6ax), 2.64-2.75 (m, 2H, H- 2eq, H- 6eq), 3.27 (br. s, 1H, OH), 3.52 (dd, 1H, J = 10.2, 5.2 Hz, H-2'), 3.60 (dd, $J=10.0,5.2$ Hz, 1H, H-1'), 3.92 (t, $J=10.0$ Hz, 1H, H-2'), 7.10 (br. d, $J=7.0$ $\mathrm{Hz}, 2 \mathrm{H}, \mathrm{ArH}) ;{ }^{13} \mathrm{C}-\mathrm{NMR}\left(\mathrm{CDCl}_{3}, 50 \mathrm{MHz}\right) \delta 11.5\left(\mathrm{CH}_{3}\right), 25.9(\mathrm{C}-5), 27.1\left(\mathrm{CH}_{3} \mathrm{CH}_{2}\right), 30.5(\mathrm{C}-$ 4), 38.3 (C-3), 52.5 (C-2), 53.1 (C-6), $59.8\left(\mathrm{CH}_{2} \mathrm{O}\right), 70.0$ (C-1'), 127.7 (C-p), 128.0 (C- o), $128.9(\mathrm{C}-\mathrm{m}), 135.4$ (C- ipso); $[\alpha]^{22}{ }_{\mathrm{D}}+15.1$ ( $0.8 \mathrm{CH}_{2} \mathrm{Cl}_{2}$ ). Anal. Calcd for $\mathrm{C}_{15} \mathrm{H}_{23} \mathrm{NO}$ : C, 77.20; H, 9.94; N, 6.00. Found C, 77.20; H, 10.04; N, 6.00\%. (-)-10: $[\alpha]^{22}{ }_{\mathrm{D}}^{-15.9}\left(c 0.9 \mathrm{CH}_{2} \mathrm{Cl}_{2}\right)$.

\section{General procedure for debenzylation reaction}

$(S)$ - and $(R)$-3-ethylpiperidine [(-)-11 and (+)- 11]. A solution of the ethylpiperidine (-)-9 or (-)$10(950 \mathrm{mg}, 4.08 \mathrm{mmol})$ in methanol- $\mathrm{HCl}(10 \mathrm{~mL})$ was concentrated to give a residue, which was dissolved in methanol $(50 \mathrm{~mL})$. The resulting solution containing $5 \% \mathrm{Pd} / \mathrm{C}(100 \mathrm{mg})$ was hydrogenated at RT until starting material disappeared in the TLC. The catalyst was removed by filtration, and the solvent was evaporated. The resulting solid was digested in $\mathrm{Et}_{2} \mathrm{O}$ to give (462 mg, 76\%) pure (-)-11 hydrochloride: ${ }^{1} \mathrm{HNMR}\left(\mathrm{CDCl}_{3}, 300 \mathrm{MHz}, \mathrm{COSY}, \mathrm{HETCOR}\right) \delta 1.06$ 
(t, $\left.J=7.7 \mathrm{~Hz}, 3 \mathrm{H}, \mathrm{CH}_{3}\right), 1.30(\mathrm{~m}, 1 \mathrm{H}, \mathrm{H}-4 \mathrm{ax}), 1.45\left(\mathrm{~m}, 2 \mathrm{H}, \mathrm{CH}_{3} \mathrm{CH}_{2}\right), 1.65-1.96(\mathrm{~m}, 2 \mathrm{H}, \mathrm{H}-$ 3ax, H- 5ax), 1.98-2.10 (m, 2H, H- 4eq, H- 5eq), 2.71 (t, $J=12.0$ Hz, 1H, H- 2ax), 2.99 (td, $J=$ 12.8, 2.7 Hz, 1H, H- 6ax), 3.38-3.48 (m, 2H, H- 2eq, H- 6eq), 3.60 (dd, J=10.0, 5.2 Hz, 1H, H1'), 3.92 (t, $J=10.0 \mathrm{~Hz}, 1 \mathrm{H}, \mathrm{H}-2$ '), 7.10 (br. d, $J=7.0 \mathrm{~Hz}, 2 \mathrm{H}, \mathrm{ArH}) ;{ }^{13} \mathrm{C}-\mathrm{NMR}\left(\mathrm{CDCl}_{3}\right.$, $75 \mathrm{MHz}) \delta 11.2\left(\mathrm{CH}_{3}\right), 28.3(\mathrm{C}-5), 27.5\left(\mathrm{CH}_{3} \mathrm{CH}_{2}\right), 29.4(\mathrm{C}-4), 36.5(\mathrm{C}-3), 45.4(\mathrm{C}-6), 49.9(\mathrm{C}-$ 2); mp 161-162 ${ }^{\circ} \mathrm{C}$; $[\alpha]^{22} \mathrm{D}-3.5$ (c $\left.1.0 \mathrm{EtOH}\right) .(-)-11:{ }^{1} \mathrm{H}-\mathrm{NMR}\left(\mathrm{CDCl}_{3}, 300 \mathrm{MHz}, \mathrm{COSY}\right.$, HETCOR) $\delta 0.88\left(\mathrm{t}, J=7.4 \mathrm{~Hz}, 3 \mathrm{H}, \mathrm{CH}_{3}\right), 0.97$ (dddd, $J=12.6,12.6,11.0,4.0 \mathrm{~Hz}, 1 \mathrm{H}, \mathrm{H}-4 \mathrm{ax}$ ), $1.17\left(\mathrm{~m}, 2 \mathrm{H}, \mathrm{CH}_{3} \mathrm{CH}_{2}\right), 1.29(\mathrm{~m}, 1 \mathrm{H}, \mathrm{H}-3), 1.42$ (qt, $\left.J=12.6,4.0 \mathrm{~Hz}, 1 \mathrm{H}, \mathrm{H}-5 \mathrm{ax}\right), 1.64(\mathrm{dm}, J=$ $12.6 \mathrm{~Hz}, 1 \mathrm{H}, \mathrm{H}-5 \mathrm{eq}), 1.83$ (dm, $J=12.6 \mathrm{~Hz}, 1 \mathrm{H}, \mathrm{H}-4 \mathrm{eq}), 1.88$ (br. s, 1H, NH), 2.20 (dd, $J=$ $11.9,10,2,1 \mathrm{H}, \mathrm{H}-2 \mathrm{ax}), 2.51$ (td, $J=12.2,2.8 \mathrm{~Hz}, 1 \mathrm{H}, \mathrm{H}-6 \mathrm{ax}), 2.99$ (dm, $J=12.2 \mathrm{~Hz}, 1 \mathrm{H}, \mathrm{H}-$ $6 \mathrm{eq}), 3.03$ (dm, $J=12.2 \mathrm{~Hz}, 1 \mathrm{H}, \mathrm{H}-2 \mathrm{eq}) ;{ }^{13} \mathrm{C}-\mathrm{NMR}\left(\mathrm{CDCl}_{3}, 75 \mathrm{MHz}\right) \delta 11.2\left(\mathrm{CH}_{3}\right), 26.7(\mathrm{C}-5)$, $27.2\left(\mathrm{CH}_{3} \mathrm{CH}_{2}\right), 31.2(\mathrm{C}-4), 38.9(\mathrm{C}-3), 47.0(\mathrm{C}-6), 52.8(\mathrm{C}-2)$; $[\alpha]^{22}{ }_{\mathrm{D}}-2.6$ (c $\left.0.68 \mathrm{EtOH}\right)$. As above, from $(+)-\mathbf{9}$ or $(+)-\mathbf{1 0},(+)-\mathbf{1 1}$ hydrochloride was obtained: $[\alpha]_{\mathrm{D}}^{22}+3.2($ c $1.0 \mathrm{EtOH}) .(+)-$ 11: $[\alpha]^{22}+2.0(c 0.68 \mathrm{EtOH})$.

$(+)-(R)$-Decarbomethoxytetrahydrosecodine. A mixture of (+)-11 (140 mg, $0.94 \mathrm{mmol}), 3-(2-$ bromoethyl)-2-ethylindole (230 $\mathrm{mg}, 0.91 \mathrm{mmol})$ and $\mathrm{NaHCO}_{3}(250 \mathrm{mg}, 2.98 \mathrm{mmol})$ in acetonitrile $(3 \mathrm{~mL})$ was heated at $80{ }^{\circ} \mathrm{C}$ for $30 \mathrm{~h}$. The mixture was cooled at RT and Et2 $\mathrm{O}$ $(100 \mathrm{~mL})$ and water $(10 \mathrm{~mL})$ were added. The phases were separated, and the organic phase was dried and concentrated. Column chromatography $\left(\mathrm{Et}_{2} \mathrm{O}\right)$ of the residue gave the alkaloid (166 mg, 64\%): ${ }^{1} \mathrm{H}-\mathrm{NMR}\left(\mathrm{CDCl}_{3}, 500 \mathrm{MHz}, \mathrm{COSY}, \mathrm{HETCOR}\right) \delta 0.85$ (qd, $J=12.0,5.0 \mathrm{~Hz}, 1 \mathrm{H}$, H- 15ax), 0.90 (t, J = 7.5 Hz, 3H, H-18), 1.24 (m, 2H, H-19), 1.27 (t, J=7.5 Hz, 3H, H-17), 1.54 (m, 1H, H- 20ax), 1.62-1.74 (m, 3H, H- 21ax, H-14), 1.80 (dm, J=12.0 Hz, 1H, H- 15eq), 1.96 (td, $J=11.0,2.5 \mathrm{~Hz}, 1 \mathrm{H}, \mathrm{H}-3 \mathrm{ax}), 2.56$ (m, 2H, H-5), 2.75 (q, J=7.5 Hz, 2H, H-16), 2.92 (dd, $J$ $=9.5,8.0 \mathrm{~Hz}, 2 \mathrm{H}, \mathrm{H}-6), 3.02-3.10(\mathrm{~m}, 3 \mathrm{H}, \mathrm{H}-3 \mathrm{eq}, \mathrm{H}-21 \mathrm{eq}), 7.05$ (td, J = 7.0, $1.0 \mathrm{~Hz}, 1 \mathrm{H}, \mathrm{H}-$ 10), 7.09 (td, $J=7.0,1.0 \mathrm{~Hz}, 1 \mathrm{H}, \mathrm{H}-11), 7.26$ (dm, $J=7.0 \mathrm{~Hz}, 1 \mathrm{H}, \mathrm{H}-12), 7.51$ (dm, $J=7.0 \mathrm{~Hz}$, $1 \mathrm{H}, \mathrm{H}-9), 7.79$ (br. s, $1 \mathrm{H}, \mathrm{NH}) ;{ }^{13} \mathrm{C}-\mathrm{NMR}\left(\mathrm{CDCl}_{3}, 75 \mathrm{MHz}\right) \delta 11.4(\mathrm{C}-18), 14.5(\mathrm{C}-17), 19.3(\mathrm{C}-$ 6), 21.7 (C-16), 25.5 (C-14), 27.5 (C-19), 30.8 (C-15), 37.9 (C-10), 54.4 (C-3), 60.2 (C-21), 60.4 (C-5), 108.8 (C-7), 110.3 (C-12), 118.0 (C-9), 118.9 (C-10), 128.5 (C-8), 135.1 (C-2), 137.0 (C$13) ;[\alpha]^{22}{ }_{D}+10.5$ (c $\left.1.0 \mathrm{EtOH}\right)$.

$(-)-(S)$-Decarbomethoxytetrahydrosecodine was obtained as above, from $(-)-12:[\alpha]^{22}{ }^{-}-10.8$ (c $1.0 \mathrm{EtOH})$.

\section{Acknowledgments}

This work was supported by the DGICYT, Spain (BQU2003-0505). Thanks are also due to the DURSI. Generalitat de Catalunya, for Grant 2001SGR-0084, the Ministry of Education, Culture and Sport for a fellowship to O. L., and the SCT of the University of Barcelona for recording the NMR and mass spectra. We thank DSM Deretil (Almería, Spain) for a generous gift of $(R)$ phenylglycine. 


\section{References}

1. (a) Amat, M.; Bosch, J.; Hidalgo, J.; Cantó, M.; Pérez, M.; Llor, N.; Molins, E.; Miravitlles, C.; Orozco, M.; Luque, J. J. Org. Chem. 2000, 65, 3074. (b) Amat, M.; Llor, N.; Hidalgo, J.; Escolano, C.; Bosch, J. J. Org. Chem. 2003, 68, 1919. For reviews, see: (c) Meyers, A. I.; Brengel, G. P. Chem. Commun. 1997, 1. (d) Groaning, M. D.; Meyers, A. I. Tetrahedron 2000, 56, 9843.

2. (a) Amat, M.; Llor, N.; Hidalgo, J.; Hernández, A.; Bosch, J. Tetrahedron: Asymmetry 1996, 7, 977. (b) Amat, M.; Pshenichnyi, G.; Bosch, J.; Molins, E.; Miravitlles, C. Tetrahedron: Asymmetry 1996, 7, 3091.

3. (a) Meyers, A. I.; Seefeld, M. A.; Lefker, B. A.; Blake, J. F.; Williard, P. G. J. Am. Chem. Soc. 1998, 120, 7429. (b) Ando, K.; Green, N. S.; Li, Y.; Houk, K. N. J. Am. Chem. Soc. 1999, 121, 5334. (c) Bailey, J. H.; Byfield, A. T. J.; Davis, P. J.; Foster, A. C.; Leech, M.; Moloney, M. G.; Müller, M.; Prout, C. K. J. Chem. Soc., Perkin Trans. 1 2000, 1977. (d) Hughes, R. C.; Dvorak, C. A.; Meyers, A. I. J. Org. Chem. 2001, 66, 5545. (e) Ikuta, Y.; Tomoda, S. Tetrahedron Lett. 2003, 44, 5931. (f) Ikuta, Y.; Tomoda, S. Org. Lett. 2004, 6, 189. (g) Brewster, A. G.; Broady, S.; Davies, C. E.; Heightman, T. D.; Hermitage, S. A.; Hughes, M.; Moloney, M. G.; Wood, G. Org. Biomol. Chem. 2004, 2, 1031.

4. Amat, M.; Llor, N.; Escolano, C.; Huguet, M.; Pérez, M.; Molins, E.; Bosch, J. Tetrahedron: Asymmetry 2003, 14, 293.

5. Previous attempts to alkylate this lactam using LDA as the base had resulted in failure: Micouin, L.; Varea, T.; Riche, C.; Chiaroni, A.; Quirion, J.-C.; Husson, H.-P. Tetrahedron Lett. 1994, 35, 2529.

6. Westrum, L. J.; Meyers, A. I. Tetrahedron Lett. 1994, 35, 973.

7. Micouin, L.; Varea, R.; Riche, C.; Chiaroni, A.; Quirion, J.-C.; Husson, H.-P. Tetrahedron Lett. 1994, 35, 2529.

8. For an alternative synthetic route to enantiopure 3-alkylpiperidines, including $(R)$-3ethylpiperidine, see: Pedrosa, R.; Andrés, C.; Duque-Soladana J. P.; Rosón, C. D. Tetrahedron: Asymmetry 2000, 11, 2809.

9. (a) Günther, A. Chem. Ber. 1898, 31. (b) Ripperger, H.; Schreiber, K.; Sych, F. J. J. Prakt. Chem. 1970, 312, 471. (c) Sakai, S.; Aimi, N.; Kato, K.; Ido, H.; Masuda, K.; Watanabe, Y.; Haginiwa, J. Yakugaku Zasshi 1975, 95, 1152. (d) Morlacchi, F.; Losacco, V.; Tortorella, V. J. Heterocyclic Chem. 1979, 16, 297.

10. Isolation: (a) Croks, P. A.; Robinson, B.; Smith, G. F. J. Chem. Soc., Chem. Commun. 1968, 1210. (b) Robert, G. M. T.; Ahond, A.; Poupat, C.; Potier, P.; Jollès, C.; Jousselin, A.; Jacquemin, H. J. Nat. Prod. 1983, 46, 694. (c) Atta-ur-Rahman; Zaman, K.; Perveen. S.; Habib-ur-Rehman; Muzaffar, A.; Choudhary, M. I.; Pervin, A. Phytochemistry 1991, 30, 1285. (d) Mroue, M. A.; Ghuman, M. A.; Alam, M. Phytochemistry 1993, 33, 217. (e) Mroue, M. A.; Euler, K. L.; Ghuman, M. A.; Alam, M. J. Nat. Prod. 1996, 59, 890. Synthesis: (f) Palmisano, G.; Santagostino, M.; Riva, S.; Sisti, M. Tetrahedron: Asymmetry 1995, 6, 1229. (g) Sakagami, H.; Samizu, K.; Kamikubo, T.; Ogasawa, K. Synlett 1996, 163. 